\title{
Un petit caillou dans la chaussure didactique : le poème
}

A little pebble in the didactic shoe: the poem

\section{Serge Martin}

\section{(2) OpenEdition}

Journals

\section{Édition électronique}

URL : http://journals.openedition.org/pratiques/4710

DOI : $10.4000 /$ pratiques. 4710

ISSN : 2425-2042

Éditeur

Centre de recherche sur les médiations (CREM)

Référence électronique

Serge Martin, « Un petit caillou dans la chaussure didactique : le poème », Pratiques [En ligne], 179-180 | 2018, mis en ligne le 31 décembre 2018, consulté le 01 mai 2019. URL : http:// journals.openedition.org/pratiques/4710; DOI : 10.4000/pratiques.4710

Ce document a été généré automatiquement le 1 mai 2019.

(c) Tous droits réservés 


\title{
Un petit caillou dans la chaussure didactique : le poème
}

\author{
A little pebble in the didactic shoe: the poem
}

Serge Martin

1 Il semble important de situer le mieux possible le défi que fait la poésie à l'enseignement, de la maternelle à l'université. On pourrait dire dans un premier temps que cela dépend du moment, du lieu, du niveau, etc. Certes les contextes, auxquels on réduit souvent les situations (voir Zask, 2008), constituent des données incontournables mais, avec la poésie, il semble assez indifférent de proposer tel poème en maternelle ou à l'université, le matin ou le soir, etc., sauf à rappeler ce qu'un enseignant peut vite apprendre à penser : qu'il est de sa responsabilité de proposer à ses élèves une programmation, c'est-à-dire tel texte, avec toute l'expérience qu'il appelle dans une pluralité situationnelle, après et avant tel autre, en regard d'une programmation élargie dans toutes les disciplines ou dans toute l'organisation pédagogique. Plus qu'une progression qui pose une maitrise avant toute expérience partagée, cette programmation devient une manière d'inciter chacune à construire des parcours qui montrent le continu des activités, des rencontres et des expériences. Par conséquent, les enseignants ont plus à penser les situations, comme rapport d'expériences, qu'à "gérer» les contextes (programmes, progressions, compétences...) surtout en poésie - ce que nous allons observer. Tout cela peut paraitre hypothétique et demande de commencer par reconsidérer la situation d'ensemble faite à la poésie dans l'enseignement puis de penser ce que seul fait un poème, l'écoute de l'écoute, jusque dans la moindre situation scolaire obligeant justement à penser situation et langage ensemble, et enfin de situer, dans cet engagement du poème, l'enjeu didactique, à vrai dire politique et éthique, de la poésie : tenir voix quand trop souvent les pertes de voix (individuelles ou collectives) ouvrent à des désubjectivations, sous les formes variées des stigmatisations (ou ce qui revient au même des identitarismes), des neutralisations ou des censures, qui réduisent l'enseignement à l'impasse des manipulations et autres impositions bien loin des inventions de savoirs et d'expériences partagés pour faire société et vivre langage. 


\title{
Trois impasses didactiques : définition, genre et réception
}

\author{
On ne se fondra pas sur des notions de bazar pour \\ étudier la poésie, on tiendra compte de ceux qui \\ ont de cette pratique une compétence. \\ (Meschonnic, 1973, p. 333)
}

2 J'aimerais commencer par signaler trois impasses quant à la didactique de la poésie et, au-delà, du français comme discipline mais aussi quant à la didactique des langues et plus décisivement du langage. Ces impasses reposent sur des progressions naturalisées généralement par des travaux savants qui constituent autant de prises de pouvoir sur la poésie. Celle-ci constitue en effet l'expérience la plus ouverte qui est offerte à tous les élèves quant aux "arts du langage » (Siouffi, 2007). Pour autant, il semble impossible d'isoler «la poésie » comme nous allons le voir dans ce qui suit, en sachant qu'elle constitue souvent à elle seule le test de tout ce qu'on fait avec tout le langage en termes de spécialisations, réductions ou instrumentalisations.

3 La première impasse est celle de la définition. La seconde, celle du genre voire de la forme. La troisième, celle de la réception. La première et la seconde sont de tout temps puisque l'essentialisme (réduction d'une œuvre à un genre en histoire littéraire ou à une forme en poétique-rhétorique) est une maladie infantile dès qu'enseignement et didactique. En effet, comme à leur principe, il y aurait cette détermination des prescripteurs à soumettre toute expérience langagière à des finalités hors langage (généralement métaphysiques ou idéologiques) ou à des principes discontinuistes (grammaire des éléments et des relations, comme si les relations ne constituaient pas les éléments). Si donc les deux premières sont anciennes, la troisième impasse est beaucoup plus récente et semble apparaitre au milieu $d u X X^{e}$ siècle en accompagnant l'individualisme post-industriel qui, paradoxalement, ne favorise pas le passage de l'individuation à la subjectivation (Caillé \& Chanial, 2011) mais entraine plutôt des formes de logiques collectives si ce n'est de collectivisme. Je les parcours rapidement pour mieux situer le défi fait par la poésie à l'enseignement, trop souvent réduit ou marginalisé voire instrumentalisé, quand bien même il reste toujours intempestif.

4 Ce défi est en effet tout à fait repéré par tout un chacun puisque, des textes officiels et prescriptifs aux pratiques formatives voire marginales souvent militantes, la "poésie " est toujours louée! Malheureusement le défi se voit régulièrement réduit à une propédeutique ou à une sortie des cadres fondamentaux, voire à un élément additionnel. Dès que «l'on fait poésie», ne parle-t-on pas plus souvent d'«initiation» que d'enseignement, plus d'«ateliers d'expression» voire de «remédiation» que d'enseignement fondamental avec les poèmes! N'a-t-on pas l'habitude de voir apparaitre régulièrement des injonctions d'enseignement supplémentaire, selon les modes ministérielles ou didactiques et selon les « actualités » (changement ministériel ou débat sociétal). Dans tous les cas, ces dispositifs qui toujours constituent comme des sorties (avant, après, ailleurs) participent à ce que rien ne change, quant au langage et aux conceptions traditionnelles et discontinuistes de ce dernier, conceptions qui continuent de gouverner les programmes et les enseignements. Cette manie de ne jamais oublier « la poésie » et de garder toujours une larme, entre déploration et grands effets de manche, désarme les acteurs de l'enseignement et constitue un leitmotiv des discours qui 
émoussent le défi de la poésie à l'enseignement. Je ne prendrai qu'un seul exemple récent : voyez la préface du ministre de l'Éducation nationale et de la Jeunesse, JeanMichel Blanquer, aux Fables de J. de La Fontaine distribuées aux élèves de CM2 pour leurs vacances avant de rentrer en $6^{\mathrm{e}}$ (donc dans un dispositif plus extra-scolaire que scolaire), qui commence par réitérer, dès son premier paragraphe, la vieille dichotomie du fond et de la forme. Cette mise au ban du défi par les pleurnicheries tient d'abord à l'impasse de la définition dans laquelle toutes les expériences avec la poésie sont sommées de répondre. Elle est bien conforme à la tradition théologico-politique qui constitue le soubassement des théories du langage de l'enseignement en France. Plutôt que de faire confiance aux protagonistes de la poésie dans l'enseignement, le contexte impose depuis presque toujours à ces derniers de répondre d'une définition de l'expérience avant son écoute, c'est-à-dire d'une surdité immédiatement engagée en regard de ce qui pourrait défaire les habitudes dogmatiques qui obligent à penser une transcendance, certes le plus souvent conventionnelle voire institutionnelle pour ne pas dire laïque, surplombant le moindre discours dès que « poésie » en classe. Qu'on parle aujourd'hui de " valeur » dans le cadre d'une " grammaire axiologique des valeurs» (Heinich, 2017) ou hier de « langage surnaturel » à distinguer de « la vulgaire prose » (article « Poésie » rédigé par F. Pécaut, théologien et premier directeur de l'école normale supérieure des jeunes filles (!) de Fontenay-aux-Roses, dans F. Buisson, 1911 - voir Martin, M.-C. \& Martin, S., 1997, p. 45 et suivantes), les expériences en poésie se voient contraintes à définir un super-objet ( la poésie » en général avec une majuscule de préférence) qui subsumerait toutes ces expériences dans une espèce d'anhistoricité ou de profondeur abyssale laissant pantois le premier sujet venu en plein subjectivisme. C'est d'ailleurs le paradoxe de cette injonction définitionnelle que de la voir, dans les contextes contemporains où l'émotion est confondue avec « la poésie » comme le romanesque avec «le roman », rejouer sans cesse, à la moindre occasion, la formule de S. Mallarmé à propos de la lune qui pointait un essentialisme auquel a contribué plus tard G. Bachelard : « elle est poétique, la garce »!

5 Mais cette injonction définitionnelle ne concernerait que les prémisses de l'enseignement, ou ses fins dans les discours maximalistes tout aussi essentialistes puisqu'ils auraient un "parfum de résurrection» (Siméon, 2015), quand l'injonction générique ou formelle toucherait au cœur des objets et démarches. Celle-ci est fondée sur la fausse étymologie de la fabrication, d'un artisanat des formes, d'un calcul des intentions ou encore d'une expérimentation des potentiels de la langue jusqu'à ses «limites». S'y inscrit une stylistique des écarts, voire une anormalité de l'expérience poétique, qui viendrait comme confirmer et la norme et le langage dit ordinaire, bref toute une politique (exclusion de certains discours) et une éthique (haut/bas langage) du langage. Une telle stylistique réitère les dichotomies traditionnelles toujours actives, où s'opposent comme des essences les Lettres et le peuple, la poésie (ou le vers) et la prose (ou le roman), l'oral et l'écrit, quand bien même depuis déjà le milieu du XIX ${ }^{\mathrm{e}}$ siècle tout ce bel édifice a été ébranlé en littérature. Il me suffit ici de citer H. Mitterand (2018, p. 125) parlant de G. Flaubert qui indique simplement ce qu'il faudrait faire avec tout poème: "Certains écrivains ont une écriture orale. Et c'est bien à cette famille qu'appartient Flaubert. Inutile d'évoquer ici le fameux "gueuloir". Il suffit de s'écouter soi-même, lisant silencieusement, pour entendre en même temps son écriture et sa voix ". Mais ne l'aurait-il pas été, ébranlé, depuis toujours, ce bel édifice séparateur, si l'enseignement comme instrument de certains pouvoirs ne venait réitérer ces formalismes qui sont sourds à l'activité de tel poème comme de telle lecture, et par conséquent à une pluralité de rapports plus qu'à des dichotomies essentialisantes qui 
aboutissent à exclure du langage nombre d'expériences poétiques et, par conséquent, à diminuer l'écoute de toutes les expériences langagières qui peuvent toutes porter leur poème.

6 On peut, à ce point de la réflexion, apercevoir combien on fait fausse route s'agissant d'une "démocratisation poétique» si l'on réitère, au cœur même de la "panoplie poétique » composée d'« outils » (Plat, 2015), de tels essentialismes génériques et/ou formalistes. Mais il semblerait qu'aujourd'hui, étant donné les difficultés d'un enseignement " massifié », on ait entièrement versé la réflexion concernant la poésie du côté de la «réception » et du «sujet lecteur » (Vibert, 2013), ou pour le moins de son rapport aux « communautés interprétatives » (Fish, 1980). Voilà donc la poésie prise dans le tournant de la "réception", lui-même rejoué par celui du «sujet» ou plutôt de l'interprétant, car la subjectivité qui préside aux subjectivations lectorales est bien toujours de l'ordre d'une activité interprétative, même s'agissant des arts du langage. L'« actualisation» (Citton, 2007 et 2010a) comme la « recontextualisation» (Ahr, 2017) constituent, dans cette optique, les opérateurs didactiques particulièrement décisifs du tournant réceptif et subjectif. Ces derniers opèrent à partir de la dichotomie fondatrice comprendre/interpréter, rejouée paradoxalement par Y. Citton (2010b, p. 135) dans le cadre de l'économie de la connaissance et du capitalisme cognitif entre connaissance et interprétation : « Et pourtant [...] c'est l'interprétation, et non la cognition, qui doit être située à la source des plus gros profits en régime de capitalisme cognitif!» - l'auteur souligne. Une telle position mime, dans un discours consensuel et autoritaire, faut-il le préciser, le débat entre philologie et herméneutique (voir par exemple, Danneberg, 1997) pour mieux manipuler les situations scolaires, et donc éviter le défi que font les poèmes et leurs lectures, quand il faudrait opposer à ce dualisme une autre conception des rapports de discours, celle des réénonciations, des reprises de parole ou énonciation continuée, c'està-dire une théorie du langage associée à des pratiques attentives aux gestes vocaux, à la voix comme relation.

7 À la suite d'une longue citation d'É. Falardeau (2003)1', S. Ahr (2017) précise très clairement ce qu'on doit entendre par "actualisation" concernant le «débat interprétatif » quand, de son côté, l'histoire littéraire serait «renouvelée » par la « recontextualisation $»$ :

Cette définition de l'interprétation permet de comprendre ce que l'institution attend des professeurs de collège comme de lycée en matière de lecture des textes littéraires : il s'agit de s'appuyer sur les significations que les élèves proposent (leur lecture actualisante du texte) pour les conduire à un travail d'interprétation, qui consistera à interroger ces significations au regard du texte et du ou des sens construit(s) ayant déjà fait l'objet d'un consensus dans le champ littéraire. Cette modalité de lecture requiert donc un espace favorisant la mise en discours et la confrontation des interprétations.

8 Toute cette philosophie habermasienne du consensus social par les arguments (Habermas, 1987), et ici du consensus scolaire, consiste en fait à opérer un tour de force permettant une manipulation assez évidente où « le jeu de l'interprétation » n'est pas si « ouvert » que cela ainsi que le demandait A. Boissinot (1998, p. 34), puisque « les conduire à un travail d'interprétation" (je souligne) suppose une ferme gouvernance interprétative. Or, in fine, cela conduit bon nombre de classes à la situation schizophrène d'une liberté première suivie par une reprise en main autoritaire, quand elle ne réussit pas par la séduction, d'autant que telle formulation, « ayant déjà fait un consensus dans le champ littéraire » (ibid.), montrerait à l'envi que le super-sujet n'est pas l'élève, ni la 
classe, mais bien «le champ littéraire ", c'est-à-dire les dominants du champ ou ses gestionnaires forcément savants! Mais ce discours fait ce qu'il peut avec la notion fondamentale d'interprétation qui est maintenue dans un cadre pseudo-démocratique quand elle est fondamentalement anti-démocratique parce que théologico-politique. Aussi, toutes les « innovations » didactiques, qui maintiendraient le régime interprétatif au principe de la lecture, ne feront au mieux que faire oublier combien ce régime est celui d'une instrumentalisation des discours, visant à faire adhérer à un sens si ce n'est à une vérité du texte et de ses lectures, c'est-à-dire à un sens du sens défini précisément par le régime culturel de l'interprétation plus ou moins institutionnalisée mais toujours naturalisée. Mais, et c'est là leur force, les œuvres peuvent, d'un point de vue démocratique, ouvrir à des énonciations fortes plutôt qu'à des interprétations qui presque toujours laissent les œuvres sans voix - et de ce point de vue la poésie en constitue le test par excellence puisqu'elle présuppose une relation vocale!

C'est très exactement ce que j'aimerais ici essayer pour apercevoir une anthropologie didactique de la réénonciation avec les œuvres - ces dernières étant avant tout des énonciations vives qui ne peuvent se contenter des habituels régimes de l'explication et de l'interprétation. C'est en effet d'abord un mouvement de refus des habitudes qui oblige à penser avec une autre tradition que celles qui ont jusqu'à ce jour occupé le terrain scolaire et universitaire dès que les œuvres sont convoquées. Voici ce que G. DidiHuberman (2018, p. 55-56) écrit contre «le ressassement du débat esthétique autour du jugement - et de la volonté critériologique qui l'accompagne presque toujours »:

Il faudrait que le philosophe ne se contente plus d'observer l'artiste depuis son retrait, sa hauteur. Qu'il ne se satisfasse plus de relever sur son calepin les éléments « sensibles » capables d'illustrer son propre monde « intelligible » déjà construit. Il faudrait que la pensée du philosophe sache répondre aux ceuvres de l'art, comme un geste répond à l'autre, comme un regard répond à l'autre, comme une caresse répond à l'autre, et par cette réponse se modifie, se déconstruit, s'ouvre tout à coup : s'élève avec la pensée-pas du danseur, s'envole avec la pensée-souffle du chanteur, s'involue avec la pensée-geste du dessinateur - pensées qui, bien sûr, ne s'arrêteront jamais à un seul organe puisqu'elles s'incarnent et se formulent esthésiquement à travers le rythme de notre chair entière, vivante et pensante.

Des répons plutôt que des réponses puisqu'il n'y a pas de questions mais bien des appels, des invites, des gestes qui demandent d'autres gestes, et ce serait le cœur de l'activité énonciative par excellence si l'on en croit É. Benveniste (1974). La formule centrale du linguiste me semble pouvoir être prise à sa réflexion sur «le langage et l'expérience humaine» qui date de 1965: «La condition d'intersubjectivité permet seule la communication linguistique ». Elle est précédée par une réflexion sur les passages de temporalités d'un locuteur à l'autre, lesquels sont considérés comme personnes, qui le conduit à ceci : « Le temps du discours n'est ni ramené aux divisions du temps chronique ni enfermé dans une subjectivité solipsiste. Il fonctionne comme un facteur d'intersubjectivité, ce qui d'unipersonnel qu'il devrait être le rend omnipersonnel » (Benveniste, 1974, p. 77). Ce passage de l'uni à l'omni que pose É. Benveniste au principe de la «condition d'intersubjectivité » constitue le fondement linguistique d'une anthropologie du langage des œuvres comme geste continué que G. Didi-Huberman me semble, dans sa situation, proposer. Je voudrais signaler que ce mouvement d'anthropologisation linguistique est initié explicitement par É. Benveniste à la fin du chapitre suivant de Problèmes de linguistique générale 2, avec une longue citation de B. Malinowski autour de la notion de communion phatique. 


\title{
Le poème, l'écoute : avec $R$. Desnos et sa « Chanson- caillou »
}

\author{
Le langage poétique est une écoute. La lecture et la \\ poétique sont l'écoute de cette écoute. \\ (Meschonnic, 1973, p. 260)
}

11 Plus largement qu'une communion phatique, que d'aucuns limiteraient à quelque aspect ou dimension, on devrait parler de relation vocale ou de voix relationnelle comme rapport de discours en trans-subjectivation, c'est-à-dire de poème comme force active d'interaction entre une forme de langage et une forme de vie. Alors, on peut examiner de plus près ce qu'il en est quant à une didactique des langues et des cultures avec les œuvres et, par conséquent, avec la poésie - il n'y aurait pas didactique spécifique autrement qu'à considérer qu'avec la poésie le test est maximal. Il s'agirait d'abord de (faire) vivre dans et par des gestes continués ces passages évoqués par É. Benveniste de l' uni à l'omni, d'une énonciation vive à une réénonciation tout aussi vive. Ce que j'appellerais les œuvres à l'œuvre ne demande pas d'organiser autre chose sous peine d'infliger aux élèves des propédeutiques qui sont des empêchements d'énonciation continuée. Dans le prolongement de ces reprises de voix, on pourrait alors penser le continu de telles expériences avec les œuvres, comme un indéchirable que G. DidiHuberman souligne, dans le passage déjà cité (2017, p. 56-57), avec les traits d'union synthématiques (pensée-pas, pensée-geste, pensée-souffle), à condition peut-être de remplacer la notion de "pensée " par trop philosophique - c'est le moins qu'on puisse dire - tout simplement par celle de parole : une parole qui fait des pas, une parole toute en gestualité, une parole qui porte un souffle autant qu'elle est portée par lui, voilà la trans-subjectivation en actes didactiques.

Un poème de R. Desnos (1999, p. 691) me permet maintenant de trouver un écho au titre de cet essai, « un petit caillou dans la chaussure didactique »:
Chanson-caillou
J'arrive en chantant
Visage de bois visage de bois
Je pars en rêvant
ô mon amour ô mon roi
Viennent les Printemps
Odeurs des bois odeurs des bois
à nous deux tuons le temps
Pour qu'il renaisse avec la joie
Eh quoi je suis toute seule
Cercueil de bois cercueil de bois
Ô mon amour ma chère gueule
Te souviens-tu de moi parfois?

13 Mais pourquoi dire "un poème "? comme si déjà une définition enserrait un discours dans une forme-genre - et ici soyons franc, ce « poème » viendrait comme heureusement permettre une "exploitation" scolaire par le format, la composition strophique et les rimes, sans compter une propédeutique facilitée par la proximité avec la chanson et ses thématiques bien acculturées où l'amour et le temps se marient depuis au moins $\mathrm{P}$. de Ronsard; bref un " poème en or » pour une didactique en «bois » qui essentialiserait la forme-genre " poème »! Mais, si l'attention advient quelque peu et n'importe quel élève 
le ferait entendre, R. Desnos a plus d'un tour pour défaire une telle langue de bois didactique, ne serait-ce qu'avec le silence trop plein du « ma chère gueule » - on pourrait facilement évoquer le tableau d'H. de Toulouse-Lautrec de 1887-1888 au titre éloquent: «La gueule de bois (la buveuse)», portrait de S. Valadon! R. Desnos titre «chanson» quand il ne titre pas « histoire » ou «conte » voire « littérature » car cette «Chansoncaillou », qui n'est donc pas une "chanson » tout court, appartient à un petit ensemble titré tardivement Les Nuits blanches. Ce titre venait initialement rassembler dans un même "cahier» (nous voilà presque dans la classe!) les notations de rêves de Youki et les poèmes de R. Desnos écrits en vis-à-vis qui avaient pour titre: "Poèmes par Youki ». D'ailleurs R. Desnos déstabilise son lecteur en renversant le cliché lyrique du poète énonciateur puisqu'ici, et souvent dans Les Nuits blanches, l'énonciation se découvre tardivement ou progressivement (le lecteur attentif peut l'apercevoir dès la fin de la première strophe avec « mon roi ») au féminin : « je suis toute seule » où l'interpellation («Eh quoi»), qui précède et qui ouvre le quatrain final, opère ainsi un double renversement de la lecture, entre ce passage du masculin attendu au féminin avec " seule ", puis l'apostrophe en apposition, plutôt dérangeante dans la langue de bois du discours amoureux, de « ma chère gueule » sous-entendu de bois.

Ce double renversement, énonciatif et thématique, que propose "Chanson-caillou » de R. Desnos, déjà lancé par son titre, serait la preuve concrète que "l'écriture est une écoute ", ainsi que H. Meschonnic (1973, p. 53) le notait dans un texte qui intervenait en regard d'une discussion avec J. Kristeva et surtout avec S. Kofman (1970) à propos de « la théorie de l'activité littéraire» chez S. Freud. H. Meschonnic, prenant appui sur l'ambivalence freudienne à ce propos, critique les présupposés d'un «vouloir-dire » et d'un non-savoir présupposés chez les écrivains par S. Kofman quand lui, H. Meschonnic, préfère repartir des propositions de S. Freud, "malgré un vocabulaire idéaliste », qui " prend l'écriture comme une écoute des signifiants, et c'est par là qu'il élimine dans sa pratique l'opposition métaphysique de la forme et du sens ", en concluant sur le fait que «la poétique est l'écoute de cette écoute» (Meschonnic, 1973, p. 53). On aperçoit alors combien la lecture de ce " poème » de R. Desnos peut engager l'écoute de l'écoute.

En effet, «Chanson-caillou » fait doublement une écoute parce qu'on sait, qu'écrit dans les marges du cahier des rêves de Youki ou se faisant passer pour un poème de celle-ci, son écriture est d'emblée une écoute résonnant d'une parole autre (voir la reproduction des pages du cahier que donne l'édition de M.-C. Dumas : Desnos, 1999, p. 676), mais aussi parce qu'il ne cesse de résonner de refrains qu'on dit populaires ou d'un ton - ce serait son côté " chanson ", qui très vite devient familier à son lecteur, tant par l'enchaînement narratif («j'arrive »/« je pars »; « renaisse »/« cercueil ») et prosodique (« de bois »/« des bois ») que par la métrique qui, même déroutante (partant d'un 5/5-5/5 pour finir sur un 4-4/4-4/4-4), tient du début à la fin le tissage des deux thèmes rimiques vocaliques /ã/ et $/ \mathrm{w} /$, que viennent perturber in fine "seule " et "gueule »... Sans oublier le fait que ce "poème » est précédé par un "Conte de fée » qui ne fait qu'écouter l'incipit de tous les contes ("Il était une fois», ibid., p. 691) quand celui qui suit, « Ô jeunesse», conte «les noces » qui «s'achèvent » ainsi : «Mais tant qu'il restera du vin dans les bouteilles / qu'on emplisse mon verre et bouchant mes oreilles / J'écouterai monter l'océan dans mon cœur » (ibid., p. 692) où l'écoute explicite est aussi celle d'une « gueule » de bois ! Par quoi, le « poème » n'est pas celui qu'on pense, ce confetti exploitable en didactique, mais une voix qui est écoute et demande qu'on l'écoute, cette écoute. 

chaussure!

\title{
Tenir voix pour faire poème : la démocratie du poème
}

\author{
Par la voix, j'entends l'oralité. Mais ce n'est plus au \\ sens du signe, où on n'entend que du son opposé à \\ du sens. Dans le continu, l'oralité est du corps- \\ langage. C'est du sujet qu'on entend. La voix est du \\ sujet qui passe de sujet à sujet. La voix fait du sujet. \\ Vous fait du sujet. Le sujet se fait dans et par sa \\ voix. \\ Meschonnic (1999, p. 149)
}

Si «l'écriture est une pratique où l'écrivain fait plus que s'énoncer. Il s'écrit » (Meschonnic, 1973, p. 183), alors, avec le poème pour le moins, la lecture est une pratique où le lecteur, écoutant cette énonciation, tient voix, comme on dit tenir tête, puisqu'il se lit/s'écrit et ne se laisse plus taire pour un sens, ou une vérité, qui n'est pas expérience d'un se dire. Tout l'enjeu pour l'enseignement de «la poésie » peut se résumer en fin de compte aux modalités de l'écoute de cette écoute qu'est le poème ("écriture » pour reprendre le mot d'époque $d$ 'H. Meschonnic). Je pars du fait que la voix du poème inventant une écoute, l'écoute de cette écoute est l'invention d'une voix qui lui ou qui en répond: en termes didactiques, on peut parler de réénonciation dont les modalités sont forcément décisives pour que les lecteurs du poème tiennent voix et ne la perdent pas en chemin, dans des détours ou attendus qui empêcheraient et l'écoute et l'écoute de l'écoute avec le poème.

18 Tout cela est délicat et demande une rupture forte avec les habitudes herméneutiques ou rhétoriques qui condamnent la voix (du poème comme écoute et de la réénonciation comme écoute de cette écoute) à s'effacer au profit d'une voix de la vérité du sens, voire de la communauté interprétative (sorte de vox dei ou vox populi), empêchant une voix libre d'advenir, ou d'une technique de contrôle des corps dans des modélisations culturelles qui arasent l'intempestivité des œuvres et des lectures. Dans le domaine de l'effacement des voix, de la maternelle à l'université, les habitudes sont rodées: les institutions et leurs prescriptions ont rarement permis une démocratie des voix même enfantines et encore moins estudiantines. On en devine rapidement les raisons : si le poème est une relation vocale où une voix advient dans et par une autre voix (Martin, 2017), " il ne trompe pas. D'où aussi peut-être le fondement physiologique de la métaphore de la “voix" » (Meschonnic, 1973, p. 183). Ce corps-langage, qu'engagent le poème et sa lecture, est généralement effacé si ce n'est nié par l'herméneutique interprétative ou la rhétorique spectaculaire, soit en réduisant l'activité du poème à celle du sens même ouvert au pont-aux-ânes de la polysémie, soit en réduisant l'activité du lecteur à celle d'une oralisation visant l'expression de ce sens. Comment faire entendre la "Chansoncaillou " sans tomber dans de telles surdités? Rien d'autre que de ne pas empêcher l'activité du poème comme écoute et d'écouter cette écoute !

Tout comme j'avais proposé de «faire œuvre avec les œuvres» (Martin, 2005), ici je proposerais, quel que soit le niveau et de manière appropriée, de ne pas laisser ce "poème " seul quand le poème est certainement plus ce qui tient ensemble Les Nuits blanches par une vocalité signifiante, dans ce qu'on pourrait appeler un atelier du dire 
ouvert par le poème. Contrairement aux habitudes, en préférant la notion de livre à celle de recueil qui oublie trop souvent le continu vocal d'un " poème » à l'autre, lire un livre de " poèmes » ne veut pas dire l'analyser, le commenter, en établir la composition, le contexte, etc., mais simplement le considérer comme un livre ouvert aux lectures en le parcourant du début à la fin, en le feuilletant par les titres, par les débuts et les fins, par les rimes, etc., c'est-à-dire en ouvrant autant de parcours que chacun'e peut s'inventer pour (se) dire sa lecture. Une consigne simple organise ces parcours individuels à partager; elle est du type: "Choisissez au moins trois éléments (titres ou rimes ou incipits ou syntagmes verbaux ou...) et classez-les du plus fort au plus faible »; ce qui ne manquera pas d'une part d'engager chacune à lire et relire, et tous à poser des jalons de l'écoute que fait le poème de R. Desnos pour apercevoir, et non établir, les nombreux passages du thématique au prosodique, de l'énonciation à la relation amoureuse, des genres populaires aux formes poétiques, etc. Ce qui revient à poser, par un rapport d'écoute, les premières hypothèses de ce que fait le poème à sa lecture, à chaque lecture, à la lecture de chacun'e.

Puis, la vocalité naturalisée du poème oblige à des activités de mise en gestes vocaux, sous des formes variées, pour que cette écoute s'entende par corps et donc par voix - ce qui est le meilleur moyen de défaire cette naturalisation vocale du poème puisque l'écoute ne cherche pas à retrouver ou à exprimer ce qui était déjà là mais à essayer ce qui est à venir avec le poème. L'exercice traditionnel de la récitation pourrait s'en trouver revisité (voir Martin, 2002) à condition qu'aucun modèle ne vienne s'imposer subrepticement ou volontairement pour forcer ces gestes vocaux à s'adapter aux formes de dictions rhétoriques trop vite plaquées, ou à exprimer une interprétation déjà là. D'aucuns rétorqueront que l'interprétation théâtrale ou simplement vocale pourrait alors advenir mais ce serait maintenir une conception représentative du dire quand ce dernier, par le passage d'un corps-langage, ne chercherait pas à lire les signifiants comme des signifiés, mais à tenter de continuer la force signifiante d'une oralité en acte. Ici, en se limitant à la seule «Chanson-caillou », il serait fort judicieux de combiner des gestes oraux relevant de la comptine autour de la rime «bois » et des gestes oraux situant la force spécifique de "gueule » qui rime avec «seule » où le désenchantement rime avec le pied-de-nez dans une demande insistante ("Te souviens-tu de moi parfois»). Ces essais de dire mettent tout en branle dans des dictions possibles, impliquant des spatialités aussi bien que des temporalités nouvelles, pour que s'entendent les silences comme les bruits du poème. On est loin de toute spectacularisation expressive, voire performance communicationnelle, autrement qu'à (se) dire pour mieux (s')écouter (dans et par) le poème. De tels essais sont à proprement parler des aventures vocales qui répondent très précisément à l'aventure de tout passage de voix que lance un poème comme écoute.

21 Toutefois, réduire les essais de dire aux dictions serait méconnaitre le continu que le poème fait entre le dire et le vivre dans toute son ampleur. C'est pourquoi j'attache la plus grande importance à l'opération continue d'une expérience d'un corps-langage qui est aussi l'expérience d'un monde qui tiendrait fortement non le rapport d'un sujet au monde (Collot, 1997) mais celui d'un sujet à un autre sujet, dans un travail du je vers l'impersonnel (qui n'est pas la non-personne), du je vers le on - de l'uni vers l'omni, pour citer É. Benveniste. Où le je ne disparait pas mais permet un passage de trans-sujet. Dans la «Chanson-caillou », il semble évident que le «bois » est ici le shiffter ou l'opérateur de glissement de cet impersonnalisation que j'appellerais un passage de voix, au moment même où «je égale on» (Meschonnic, 1973, p. 55). Aucune complication mais tout 
simplement le «bois » ici demanderait une courte réalisation documentée proposant, chacune à sa manière, un "documenteur » (aucun objectif véridictionnel dans cette documentation) séquentiel ou panoramique qui associerait dans un sujet-monde " visage de bois ", « odeurs de bois ", «cercueil de bois » et «gueule »... Mais ce serait par trop s'arrêter au "poème " quand le poème-Desnos demanderait de poursuivre le ton «comptine » et plus largement la tonalité «populaire» que construit le livre Les Nuits blanches : sans du tout poser des intertextualités et autres rapports d'objets désubjectivés, il s'agirait de montrer la voix continuée d'expériences réénoncées, chansons et gestes, comptines et histoires, comme autant de corps-langages, qui du "Conte de fée " à l'«Histoire du petit bossu» (Desnos, 2011, p. 692-693) en passant par «Histoire de Folfanfifre » (ibid., p. 684-685) mais aussi « Bonjour bonsoir » (ibid., p. 683-684) offrent des sémantiques sérielles, bien plus que des thèmes, qui font là encore comme un glissement du je au on: «Amour toi qui conduis nos gestes » (ibid., p. 693)!

Mais il y aurait, du cœur de la lecture avec les poèmes, à tenir voix. Après avoir tenté la reprise des indices d'une lecture et donc d'une écoute, puis développé des essais de voix résonnant de cette écoute et, enfin, après avoir visé une dépersonnalisation comme transsubjectivation à l'œuvre, on ne peut oublier que le lecteur du "poème", pour que le poème continue, se doit encore une fois d'écouter monter une voix répondant à la voix écouteuse du poème, comme s'il se découvrait tenir voix. Rien de mieux alors que de déconfondre le je et le moi à même le "poème" (étant bien entendu que cela peut éventuellement s'étendre aux autres "poèmes» des Nuits blanches) en essayant des passages de voix plutôt que des identités vocales car « la voix est relation » (Meschonnic, 1982, p. 294; Martin, 2017) : comment ne pas suggérer de faire entendre, dans des phylactères, les paroles possibles des "visages de bois ", des " odeurs des bois » et bien évidemment du « cercueil de bois » et pourquoi pas de «ma chère gueule »... de bois. Ces résonances en je inventent alors autant de masques qui peuvent faire résonner des possibles vocaux et ainsi continuer la voix qui écoute du poème par les voix qui écoutent cette écoute des lectures.

En conclusion, le défi que fait la poésie à l'enseignement, de la maternelle à l'université, ne serait pas celui de sa définition : des comptines aux poèmes en prose en passant par le vers libre et toutes les modalités culturelles que construisent les arts du langage, des chansons à la musique verlainienne, ou de la «crise de vers" (S. Mallarmé) aux performances sonores, de Villon au slam. Le défi ne serait pas plus celui d'un genre qui, soit ne tiendrait pas dans la discipline français et se perdrait dans les arts (danse, théâtre, scènes...), soit se réduirait à une peau de chagrin, à une stase culturelle, et laisserait s'échapper les expériences qui comptent. Le défi ne serait pas plus celui d'une réception qui depuis bien longtemps a vu la poésie s'épancher dans les moments hors cadres normatifs, ou festifs ou générationnels, puis disparaitre pour passer aux choses sérieuses tant quotidiennes que vitales, scolaires et socialement rentables. Ayant traversé les rénovations structuralistes et post-modernes, ayant été porté par les jeux linguistiques autant que par les libérations subjectivistes, la poésie thématisée, mise en fiches ou pratiquée en sauvetage social, resterait comme un petit caillou dans la chaussure usée d'une didactique cheminant des compétences langagières aux épreuves des concours de Lettres modernes, en passant par les ressources pour les voies professionnelles.

24 Le défi est bien celui d'une orientation forte, que la poésie oblige à construire poème par poème, par les poèmes au cœur de l'enseignement du français (Martin, 2010) : celle du continu poétique, éthique et politique d'une didactique de la relation vocale (forcément plurielle) 
dont le poème comme expérience (Dewey, 2010) constitue le levier décisif. Ce que l'enseignement (ses institutions comme ses acteurs) fait de la poésie est alors un test de ce qu'il fait du langage, du sujet, de la société. Test de l'écoute (ou non) des vies comme de la démocratie des voix. On ne peut alors s'arrêter de montrer, au quotidien des classes comme au quotidien de la pensée didactique, comment avec les poèmes, il s'agit de tenir voix, comme on dit tenir tête, pour que le discontinu didactique n'épuise pas et les valeurs et les acteurs d'un enseignement démocratique.

\section{BIBLIOGRAPHIE}

AHR, S. (2017). «Actualisation/(re)contextualisation des textes patrimoniaux : d'un questionnement épistémologique à des questions d'ordre didactique ». Recherches et travaux 91. En ligne : http://journals.openedition.org/recherchestravaux/954.

BENVENISTE, É. (1974). Problèmes de linguistique générale 2. Paris : Gallimard.

BoIssinot, A. (1998). Littérature et Histoire. Paris : Bertrand-Lacoste.

BUISSON, F. (1911) Nouveau Dictionnaire de pédagogie et d'instruction primaire. En ligne : http:// www.inrp.fr/edition-electronique/lodel/dictionnaire-ferdinand-buisson/document.php?id=3405. CAILlÉ, A. \& CHANIAL, P. (2011). « Présentation ». Revue du MAUSS 38, p. 5-30. En ligne : https:// www.cairn.info/revue-du-mauss-2011-2-page-5.htm.

CITTON, Y. (2007). Lire, interpréter, actualiser. Pourquoi les études littéraires ? Paris : Amsterdam.

CITTON, Y. (2010a). «Économie de la connaissance ou cultures de l'interprétation? ».

L'Observatoire, la revue des politiques culturelles hors-série 3, p. 36-42.

CITTON, Y. (2010b). L'avenir des humanités. Économie de la connaissance ou cultures de l'interprétation? Paris : La Découverte.

Collot, M. (1997). La matière-émotion. Paris : Presses universitaires de France.

DANNEBERG, L. (1997). « Philosophie contre philologie Herméneutique philosophique et études littéraires ». Trad. par O. Agard. Revue germanique internationale 8, p. 31-46. En ligne : https:// journals.openedition.org/rgi/638.

DESNOS, R. (1999). CEuvres. Paris : Gallimard.

DEWEY, J. (2010) [1934]. L’Art comme expérience. Trad. de l'anglais (États-Unis) par J._P. Cometti et al. Paris : Gallimard.

DIDI-HUBERMAN, G. (2018). Aperçues. Paris : Minuit.

FALARDEAU, É. (2003). « Compréhension et interprétation : deux composantes complémentaires de la lecture littéraire ». Revue des sciences de l'éducation 29 (3), p. 673-694. En ligne : https:// www.erudit.org/fr/revues/rse/2003-v29-n3-rse966/011409ar/.

FISH, S. (2007) [1980]. Quand lire c'est faire. L'Autorité des communautés interprétatives. Trad. de l'anglais (États-Unis) par É. Dobenesque. Paris : Les Prairies ordinaires. 
HABERMAS, J. (1987) [1981]. Théorie de l'agir communicationnel 2 tomes. Trad. de l'allemand par J.M. Ferry. Paris : Fayard.

HEINICH, N. (2017). Des valeurs. Une approche sociologique. Paris : Gallimard.

KofMAN, S. (1970). L'enfance de l'art. Une interprétation de l'esthétique freudienne. Paris : Payot.

martin, M.-C. \& MARTin, S. (1997). Les Poésies, l'école. Paris : Presses universitaires de France.

MARTIN, S. (2002). « Faire récitation, faire poésie, produire un poème : chercher le ton ou chercher la voix? ». Cahiers Robinson 11, p. 81-97.

MARTIN, S. (2005). «Faire œuvre avec les œuvres ». Le français aujourd'hui 149, p. 67-73. En ligne : https://www.cairn.info/revue-le-francais-aujourd-hui-2005-2-page-67.htm.

MARTIN, S. (2010). «Présentation. Les poèmes au cœur de l'enseignement du français ». Le français aujourd'hui 169, p. 3-14.

MARTIN, S. (2017). Voix et relation. Une poétique de l'art littéraire où tout se rattache. Taulignan :

M. Delabre.

MEsChONNIC, H. (1973). Pour la poétique II. Epistémologie de l'écriture. Poétique de la traduction. Paris : Gallimard.

MESCHONNIC, H. (1982). Critique du rythme. Lagrasse : Verdier.

MESCHONNIC, H. (1999). Poétique du traduire. Lagrasse : Verdier.

MITTERAND, H. (2018). « À la recherche du rythme ». Europe 1073-1074, p. 123-135.

PLAT, J. (2015). « Méthodologies et outils de la lecture : rhétorique, poétique, stylistique, théorie littéraire ». En ligne $:$ http://litt-arts.univ-grenoble-alpes.fr/fr/presentation/axes-de-recherche/ methodologies-et-outils-de-la-lecture-rhetorique-poetique-stylistique-theorie-

litteraire-62727.kjsp.

SIMÉON, J.-P. (2015). La Poésie sauvera le monde. Paris : Le passeur.

Siouffi, G. (2007). « Du sentiment de la langue aux arts du langage ».

Ela. Études de linguistique appliquée 3 (147), p. 265-276. En ligne : https://www.cairn.info/revueela-2007-3-page-265.html.

VIBERT, A. (2013). « Faire place au sujet lecteur en classe ». En ligne : http://eduscol.education.fr/ lettres/im_pdflettres/intervention-anne-vibert-lecture-vf-20-11-13.pdf.

ZASK, J. (2008). « Situation ou contexte ? Une lecture de Dewey ». Revue internationale de philosophie 245, p. 313-328. En ligne : https://www.cairn.info/revue-internationale-de-philosophie-2008-3page-313.htm.

\section{NOTES}

1. «Si la compréhension est construction du sens à partir des éléments explicites et implicites du texte, l'interprétation sera spéculation sur le "pluriel du texte" (Canvat, 1999, p. 103), et exploration herméneutique. Et comme la spéculation et l'exploration n'appartiennent plus au domaine du consensus explicatif vers lequel tend la compréhension, l'interprétation poursuivra plutôt une "signification", qui renvoie étymologiquement à l'action d"indiquer", de choisir parmi tous les possibles signifiants. Si le sens est en partie intrinsèque au texte, la signification en est extrinsèque, créée par un lecteur interprète qui cherche à produire de nouveaux signes à 
partir de ceux qu'il perçoit dans le texte. [...] L'interprétation passe obligatoirement par une confrontation sociale qui lui conférera sa légitimité. Elle est l'actualisation d'une signification par un sujet au sein d'une communauté, dans la mesure où elle participe à la sémiosis ininterrompue.»

\section{RÉSUMÉS}

Comment ne plus se laisser prendre par les injonctions traditionnelles de la définition ou du genre et par celles plus récentes de la réception, pour libérer l'écoute de l'écoute qu'est tout poème comme passage de voix ? Tel serait la problématique de cet article qui, à partir d'un poème de R. Desnos, "Chanson-caillou », et du livre dont il est issu, Les Nuits blanches, cherche à faire œuvre avec les œuvres, c'est-à-dire à ce que les élèves et leur professeur tiennent voix dans et par les poèmes au cœur de l'enseignement du français. Il ne s'agit donc pas d'une didactique clé en main mais d'une recherche de l'écoute du langage comme activité trans-subjective par un essayer dire qui n'est pas sans implication politique pour un enseignement démocratique où chaque voix compte.

How can one no longer let oneself be caught by the traditional injunctions of definition or genre and by those more recent of the reception, to free the listening of the listening which is any poem like passage of voices? Such would be the problematic of this article which, based on a poem by R. Desnos, "Chanson-caillou", and the book from which it came, Les Nuits blanches, seeks to work with works, that is to have students and their teachers hold a voice in and through the poems at the heart of French teaching. It is not therefore a turnkey didactic but a search for listening to language as trans-subjective activity by trying to say that is not without political implication for a democratic education where each voice counts.

\section{INDEX}

Keywords : poem, listen, diction, voice, subjectivation, essay

Mots-clés : poème, écoute, dire, voix, subjectivation, essai

\section{AUTEUR}

\section{SERGE MARTIN}

Université Sorbonne-Nouvelle - Paris 3, DILTEC, EA 2288, Université Sorbonne-Nouvelle - Paris

3/CNRS, THALIM, UMR 7172, f-75005, France 